Voix et Images

\title{
Une esthétique de la lutte : l'écriture de Philippe Haeck
}

\section{Max Roy}

Volume 6, numéro 3, printemps 1981

Philippe Haeck

URI : https://id.erudit.org/iderudit/200280ar

DOI : https://doi.org/10.7202/200280ar

Aller au sommaire du numéro

Éditeur(s)

Les Presses de l'Université du Québec

ISSN

0318-9201 (imprimé)

1705-933X (numérique)

Découvrir la revue

Citer cet article

Roy, M. (1981). Une esthétique de la lutte : l'écriture de Philippe Haeck. Voix et Images, 6(3), 381-392. https://doi.org/10.7202/200280ar d'utilisation que vous pouvez consulter en ligne.

https://apropos.erudit.org/fr/usagers/politique-dutilisation/ 


\title{
Une esthétique de la lutte: l'écriture de Philippe Haeck
}

\author{
par Max Roy
}

Dès le siècle dernier, les conceptions de l'art ont commencé à être ébranlées au profit d'une libéralisation de ses structures et des valeurs qu'il est appelé à supporter. L'artiste s'est vu progressivement investi d'un pouvoir inédit qui définit le rapport de l'œuvre à son milieu. En réaction contre des notions idéalistes telles la transcendance et l'inspiration, la littérature a acquis une fonction sociale de plus en plus marquée du signe de la contestation, et un fonctionnement de moins en moins secret. L'institution littéraire ${ }^{1}$ admet désormais dans ses rangs le plus et le moins: les courants progressistes et traditionnels coexistent avec parfois autant de bonheur ${ }^{2}$.

Depuis ses premières interventions dans le milieu littéraire québécois, Philippe Haeck n'a cessé de contester le caractère gratuit, la fonction d'exutoire individuel de l'écriture bourgeoise. A la pseudo-neutralité comme à la beauté pure, il oppose l'effet foncièrement actif de l'écriture moderne. Ainsi la jouissance privée, l'expérience du sublime et l'activité purement intellectuelle doivent faire place au plaisir partagé, au quotidien valorisé et à l'intervention du physique. Du coup, l'écrivain prône une contribution importante de l'art à I'histoire de notre société, à son devenir plus humain surtout. Il engage l'écriture au sens critique, à l'éducation de même qu'à l'entreprise utopique de la révolution permanente ${ }^{3}$. Le projet révolutionnaire reste néanmoins limité à "la parole d'écriture, parole de contestation incessante ${ }^{4}$, et appelle une critique de la notion de poésie 5 .

La poésie n'a rien à voir avec des chants plus ou moins heureusement rimés ou rythmés qui flattent les choses et les êtres bien en place et les laissent à leurs places ${ }^{6}$.

L'engagement de l'écrivain, aussi sincère qu'il soit, s'inscrit dans sa pratique de l'écriture et n'implique pas nécessairement d'autres formes de militantisme. Loin de signifier la primauté de l'abstraction théorique sur l'action concrète, le vouloir-faire de l'auteur reste insaisissable ailleurs que dans son discours. C'est son discours poétique qui nous intéresse ici. ${ }^{7}$

La poésie reçoit chez Haeck une fonction éminemment critique. Elle n'échappe pas pour autant à la fonction esthétique. C'est cependant une référence moins subjective qu'historique qui présidera à la définition du beau: 
la beauté aujourd'hui est dans la lutte des classes des individus des peuples des races des sexes opprimés contre les classes les individus les peuples les races les sexes oppresseurs

la poésie naît du côté de la beauté, la poésie est du côté de la lutte ${ }^{8}$

L'enjeu poétique se double de l'enjeu politique. Le rapprochement des activités n'est évidemment pas neuf et il a même toute une tradition au Québec. ${ }^{9}$ Dans les courants nationalistes conservateurs ou progressistes, dans les manifestations du terroir ou de l'automatisme, chez Parti pris ou aux Herbes rouges, l'art a toujours été lié à quelque chose. C'est un lieu commun que le langage n'est jamais innocent, qu'il traduit toujours une origine; le nonalignement n'est au mieux qu'une volonté de neutralité ou d'indépendance et risque fort de devenir à son insu un assentiment à l'ordre établi. Il n'y a pas de situation apolitique. Philippe Haeck et toute une génération d'écrivains ont délibérément pris le parti d'un langage poétique inscrit à l'enseigne marxiste car c'est encore de nos jours la lecture de l'histoire la plus prégnante, qui suggère un mode de pensée et d'action accordé aux nécessités de l'évolution. L'esthétique proposée par Haeck tient compte de la problématique de la lutte des classes, ce qui en fait la réalité centrale:

\section{Il faut que le texte de fiction travaille à la dictature du prolétariat. ${ }^{10}$}

Comme le montre une citation précédente, la lutte portera également sur des questions individuelles, nationales, raciales, sexuelles. Féminisme et nationalisme semblent côtoyer socialisme et humanisme. Projet fort généreux que celui-là où l'apparence des contradictions le cède en importance à une soif de liberté et d'amour: "la lutte continue pour que la vie naisse, l'amour»." D'une conception d'abord sociale, l'écriture devient une esthétique de la lutte. La poésie renferme à la fois un potentiel de plaisir et de subversion. Voilà du moins ce que lui prête Philippe Haeck.

Dans l'Action restreinte, De la littérature, le texte est défini comme le produit d'un montage:

Un texte est toujours un montage, un assemblage d'éléments divers: événements, discours, écritures, blancs. Tout passe dans le texte, toute ma (la) vie, c'est pourquoi l'écriture est complexe. Dès que je sais lire, écrire, rien n'est simple, tout se renvoie, les associations sont infinies: c'est le grand jeu. ${ }^{12}$

Le texte rend ainsi le vécu, emmêlé, multiple et changeant, dans ses aspects de monotonie ou de surprise. L'esthétique pratiquée par Philippe Haeck dans sa poésie consiste donc dans la conjugaison de plusieurs discours, dans la technique de la «greffe textuelle» ${ }^{13}$. C'est la manière moderne de brancher l'écriture sur la réalité, car "il n'y a pas la vie et les livres».14 Pour rendre l'impression du vécu qui, à l'instar de l'histoire, n'est jamais univoque, le texte trame plusieurs fils (le poète emploie cette image dans la description de son activité). L'examen des faits nous permet de distinguer quatre types de segments discursifs : descriptif, narratif, théorique et didactique. Cette typologie est fonction de l'œuvre particulière à l'étude et peut s'avérer insuffisante pour 
un autre corpus; elle ne prétend pas à l'universalité. Le premier critère de discrimination repose évidemment sur le contenu du discours. Le second a trait à l'importance de la fonction de communication, qui va du moins au plus. Ainsi, le discours descriptif ne commande pas l'adresse au destinataire tandis que le discours didactique n'admet pas son absence. Par ailleurs, le discours théorique (ou critique) fait généralement apparaître le destinateur alors que le narratif a tendance à le dissimuler. Il faut dire enfin qu'un segment trouve sa limite avec l'apparition d'un autre type de discours.

Si, dans Nattes, Philippe Haeck affirme le «refus de la description»15, il écrit ailleurs: «beaucoup de descriptions sont encore nécessaires pour tromper l'aveuglement défendu par quelques écrivains».16 Les passages descriptifs occupent une place importante dans son discours poétique et côtoient volontiers la relation d'événements plus ou moins banals. Dans l'exemple qui suit, les discours descriptif et narratif s'enchaînent:

Du ciment. Du goudron. Des madriers. Du contreplaqué. Ainsi chargés les journaliers marchent sur les toits, montent dans des échelles, ont le vertige à quel étage. ${ }^{17}$

Le discours théorique ne sera pas moins important chez Haeck. Sous cette catégorie entrent les segments sui-réflexifs, métalinguistiques, portant sur l'acte d'écrire, l'engagement de l'écriture, la lutte à mener, la stratégie à adopter. Ce discours peut déboucher sur le mot d'ordre, dans lequel cas il devient didactique. Ce dernier type enseigne, exhorte a l'action, invective le lecteur et dicte son comportement aussi bien social qu'intellectuel. Le thérique et le didactique se suivent dans cet extrait :

Quand tu parles, écris, il y a avec les mots que tu utilises toutes leurs histoires; sonde ces histoires pour t'assurer que les mots ne pensent pas à ta place. ${ }^{18}$

La coexistence de ces types de discours et leur combinaison variée contribuent à faire sentir le quotidien, la spontanéité, l'influence du concret sur l'intellect et vice versa. Philippe Haeck se livre tout entier dans son écriture; il nous raconte ses réactions d'écrivain face à son propre travail. II brosse un tableau à la fois trivial et inaccoutumé de la vie en train de se faire. Dans cette perspective, la beauté - notion plus impressionniste que réaliste et plus subjective qu'objective - correspond tout simplement à la vie redécouverte dans sa plénitude, à la lutte pour cet épanouissement. S'il est impossible de réduire cette rhétorique au seul reflet parce que l'art comporte nécessairement une vision critique de la réalité, il faut néanmoins interroger la notion de réalisme. ${ }^{19}$

La notion de Réalisme, trop souvent étudiée en soi, ne peut se comprendre que dans l'évolution historique des littératures; le Réalisme n'est, à chacune de ses apparitions, qu'une volonté de retrouver l'humanité réelle dont s'est écartée la littérature d'inspiration livresque. ${ }^{20}$

Au-delà de la question philosophique qu'elle soulève, le statut foncièrement fictif du langage nous oblige à tenir cette notion pour un effet et à en rappeler le caractère illusoire. Elle a tout de même une présence dans le 
discours, soit dans sa structure soit dans son objet, pour autant que son contexte historique soit suffisamment reconnaissable. L'illusion réaliste tient à un effet de lecture rendu possible par un univers (de l'histoire et/ou de langage) commun entre l'auteur et le lecteur et les marques de cet univers dans le texte. II y a autant de réalismes qu'il y a de pactes littéraires établis entre des producteurs et des consommateurs de textes. En effet, si la référence joue habituellement dans l'œuvre engagée un rôle constitutif, il existe une variété infinie d'esthétiques de la représentation. Balzac, Zola, Sartre et Robbe-Grillet, pour ne nommer que quelques écrivains français, sont réalistes de manières fort différentes. Le discours critique a fait de la notion de réalisme un véritable nœud d'ambiguïtés: «an exceptionally elastical term» selon J.A. Cuddon. ${ }^{21}$ Le terme désigne tantôt un style tantôt un contenu. On s'accorde pour dire qu'il tient plutôt du matérialisme, ce qui marque son appartenance idéologique mais ne nous donne pas un critère de classement des unités du discours. A l'instar de Brecht, Philippe Haeck oppose le réalisme au formalisme, situant le débat au niveau de l'expression. Par ailleurs, à la tradition critique qui fait du romantisme sa contrepartie, il rétorque:

LES VRAIS REALISTES SONT ROMANTIQUES: ILS DEBORDENT L'ORDRE ÉTABLI, ILS VOLENT DÉJA AU-DESSUS DU MONDE TEL QU'IL EST, ILS SONT DEJA DANS LE MONDE TEL QU'IL DEVIENT. ${ }^{22}$

La subjectivité trouve sa pertinence dans l'écriture moderne; elle n'entrave pas la lutte: « je n'est pas un mot obscène ». ${ }^{23}$ Au contraire. JE préside à la dénonciation, à la critique; il assure la véracité de l'expression et confère au réel objectif une profondeur de signification. Dans une formule plutôt étriquée, l'écrivain résume ainsi sa conception du réalisme:

Le réalisme. Courage de dire sa vérité. Fantômes et luttes. Le trop de la beauté. Ravissement. Emportement. ${ }^{24}$

Ce n'est donc pas à la seule représentation du réel objectit que nous convie cette esthétique qui, pourtant, loin de nier le sens de l'histoire, veut en accélérer la marche. L'écriture de Philippe Haeck vise en définitive sinon la synthèse, du moins la coexistence de l'objectif et du subjectif, de la réalité extérieure et de la passion, de l'histoire et du sacré:

L'avant-garde se divise en deux: du côté du sacré le plaisir formaliste, le rituel littéraire (Baudelaire, Flaubert, Mallarmé), et l'attention aux questions métaphysiques, la mort avant tout; du côté de l'histoire l'écoute des voix (Woolf, Duras) et des masses (Michelet, Hugo, Brecht). S'il y a un conflit entre les tenants du sacré - au-delà de l'histoire même quand ils en parlent - et les tenants de l'histoire - rejet de la théorie de l'art pour l'art, du culte littéraire où individus et peuples ne sont plus que sonorités qui jouent dans une syntaxe - l'écrivain réaliste prend parti pour l'histoire. Mais ce conflit peut être vécu autrement: tenter à l'intérieur d'une écriture d'amalgamer le sacré, la force de l'écriture, et l'histoire, les effets de l'écriture, autrement dit la fin et le début de I'histoire. Combiner Brecht et Mallarmé. ${ }^{25}$

Cette combinaison du sacré et de l'histoire définit le projet d'écriture de Philippe Haeck et permet l'insertion du désir dans l'histoire. La poésie répond 
à la passion de la vie en associant dans une même problématique les motivations personnelles et collectives contenues dans les termes désir et révolution. Les finalités du plaisir et de la lutte, servies par un pareil mariage. nous renvoient à la pragmatique et appelleraient sans doute un long commentaire sur le rôle et l'importance de la poésie dans l'institution littéraire, sur ses protagonistes, sur son efficacité. Nous nous en tiendrons pour l'instant à la dialectique inscrite dans la praxis poétique de Polyphonie que l'auteur décrit de la façon suivante:

Polyphonie. L'affirmation du je contre l'ordre bourgeois qui normalise - production en série de l'individu moyen - et contre la pensée de gauche qui considère peu importants les éléments subjectifs - suppression au nom de la lutte des classes des conflits individuels. 26

Chose intéressante à savoir, Philippe Haeck avait d'abord songé à intituler l'ouvrage Pour la révolution. ${ }^{27}$ En somme, Polyphonie reflète la dialectique désir-révolution, médiatisée par l'activité de l'écriture. On remarquera l'étroite correspondance des termes avec le modèle triadique élaboré par le Groupe $\mu v$ pour l'étude du poème. ${ }^{28} \mathrm{Ce}$ modèle pèche par sa trop grande généralité, faisant du rapport de l'écriture (logos) à l'homme (anthropos) et au monde (cosmos) le principe de l'écriture poétique. Cette réserve faite, la comparaison reste féconde et donne la figure suivante:

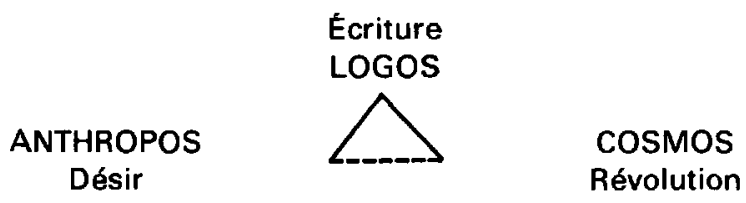

Ce qu'il importe surtout de souligner, c'est, avant même l'actualisation de la dialectique désir-révolution, la double personnalité de la poésie, fondée sur un rapport au sujet (à l'être) et sur un rapport à l'objet (au faire). II en résulte deux types d'écriture. A la dualité subjectif/objectif qui a l'inconvénient de faire croire à l'exclusion de l'humain du discours descriptif ou à l'impossibilité d'une prise en charge du réel par l'autobiographie par exemple, on préférera l'antonymie lyrique/prosaïque. Polyphonie possède ces deux registres. Le discours poétique (le texte entier) peut donc être lu selon cet aspect. On s'apercevra d'ailleurs, à l'occasion, de la conjonction de ces registres, ce qui méritera une attention toute spéciale.

Les marques discursives de ces deux registres existent aux plans de l'expression et du contenu. ${ }^{29}$ Le discours rapporté - qui est parfois marqué par les guillemets - est une motivation prosaïque au plan de l'expression tout aussi significative et agissante sur la lecture que la description la plus fidèle possible du lieu où se tient ce discours. C'est la recherche de l'illusion réaliste autant au niveau du style qu'à celui de l'objet. Au plan du contenu, le registre prosaïque donne priorité aux lieux, événements, faits objectifs, réalités matérielles ou intellectuelles concrètes; au plan de l'expression, c'est la simplicité du vocabulaire, de la syntaxe, le sens propre, les constructions 
usuelles, la lisibilité. La perception qu'en fait la lecture est rassurante. Elle a l'impresssion d'être en terrain connu, dans le domaine du préconstruit.

A l'opposé le registre lyrique dont le contenu rélève de la fiction, d'un univers imaginaire où les choses possèdent des traits inédits ou inhabituels. Son expression est généralement recherchée, imagée, soignée, inattendue. On peut l'appeler, par convention, symbolique. La lecture risque d'être plus difficile, plus inquiète; elle peut procurer plus de plaisir aussi. C'est le domaine du construit.

Dans le discours poétique en général, les registres prosaïque/lyrique ne sont pas exclusifs. Théoriquement liés, le contenu et l'expression peuvent différer par leurs formes et substances qui, elles, restent indissociables. Ce phénomène, qui a sans doute sa pertinence idéologique et pratique, a son mode d'existence dans Polyphonie. A un contenu prosaïque peut correspondre une expression lyrique. C'est le cas de ce segment:

la joie joyeuse de t'aimer $A$, de t'aimer $O$, il n'y a pas à dire, je t'aime, je t'hème, je t'haime, je t'èm, je t'aim ${ }^{30}$

L'inverse est également possible - on verra qu'il est plus fréquent chez Haeck - où le contenu tient de la fiction, d'une relation entre les choses réalisée par l'imagination, alors que l'expression utilise une forme usuelle:

l'écriture est un système de caresses. ${ }^{31}$

En somme, les registres prosaïque et lyrique appellent des attitudes de lecture différentes. L'accès plus ou moins facile au texte, la réponse à l'attente de limpidité ou de surprise et la complicité tacite entre les partenaires de la communication comptent parmi les effets du système, que le tableau 1 veut illustrer:

Tableau 1

\begin{tabular}{|l|l|l|}
\cline { 2 - 3 } \multicolumn{1}{c|}{} & \multicolumn{2}{c|}{ REGISTRES DU DISCOURS } \\
\cline { 2 - 3 } \multicolumn{1}{c|}{} & PROSAIIQUE & LYRIQUE \\
\hline EXPRESSION & Usuelle & Symbolique \\
\hline CONTENU & Perceptible & Imaginaire \\
\hline
\end{tabular}

La description minutieuse d'un paysage, la relation chronologique d'un événement, les dialogues rapportés, le monologue intérieur et les explications psychologiques même, sont, à des dégrés divers, des techniques de vraisemblance. Elle suggèrent au lecteur de croire ce qu'il lit. En apparaissant dans le texte sous le registre prosaïque, elles contribuent à l'illusion réaliste. Une tout autre façon d'exprimer la réalité extérieure consiste précisément à dénoncer le caractère fictif de la langue et de la littérature, en rappelant au lecteur qu'il est en train de lire un texte, que ce texte est le produit d'un travail, 
que ce texte affirme l'écrivain-auteur. Tomachevski remarquait déjà en 1925 que:

la négation du caractère littéraire de l'œuvre dans l'œuuve elle-même est une expression de la motivation réaliste que nous rencontrons fréquemment. ${ }^{32}$

Cette attitude s'inscrit dans le procès de l'énonciation. Elle atteste les déterminations historiques et signale, de manière optimale, la relation entre l'écriture et la lecture, entre la création littéraire et le réel, entre le logos et le cosmos. Polyphonie marque singulièrement de cette façon son rapport à la réalité. Un exemple suffira parmi des dizaines possibles:

Je mange lentement des jujubes, ma pensée occupée à cette écriture à livrer à celles et à ceux que j'aime, à la révolution à mener. Combien de fois aurais-je repris ce texte. J'attends tout du travail des femmes et des hommes avec qui je vis. ${ }^{33}$

Notre modèle hypothétique de description serait inopérant s'il négligeait de distinguer le procès de l'énonciation et le procès de l'énoncé. Le tableau 1 doit être bissé puisque l'énonciation et l'énoncé ont un fonctionnement indépendant.

En intégrant la typologie des discours suggérée précédemment à ce modèle, nous obtenons un instrument fort utile pour interroger le texte de Polyphonie. Pour les besoins de la démonstration, l'étude se limite à trois extraits significatifs: a) le tout premier texte de l'ouvrage intitulé *le poète dit...»34; b) «l'improvisation»35; c) le premier texte de $*$ Car tendresse (la dernière partie de Polyphonie), commençant par les mots «des chiens» ${ }^{36}$. Les données de l'analyse apparaissent rassemblées dans le tableau 2. Notons, pour l'intelligence de ce tableau, que l'absence d'une catégorie vaut une absence de marque et que sa présence est évaluée selon trois degrés: minimale $(x)$, redondante (xx) et insistante (xxx). Nous identifions l'intensité des phénomènes selon un ordre proportionnel.

Ce tableau exprime bien la prééminence du registre prosaique dans l'écriture de Philippe Haeck. Sous ce registre, comme au total d'ailleurs, les discours descriptif et narratif dominent, suivis de très près par le discours théorique. Ce dernier, est presque autant assumé par l'énonciation que par l'énoncé. Dans l'ensemble, le procès de l'énonciation est beaucoup moins marqué que le procès de l'énoncé. L'emploi restrictif de ces notions y est évidemment pour quelque chose: il ignore, par exemple, la disposition graphique et le découpage textuel qui signalent, au-delà du code, l'énonciation. Bien que significatifs, de tels phénomènes ne sont pas déterminants sur la lecture. Si le texte peut théoriquement accuser son énonciation de manière lyrique, il appert que l'écriture à l'œuvre dans Polyphonie se manifeste plutôt de façon prosaïque: «le poète dit», «reprenons tout», etc. Par ailleurs, le discours didactique - qui est mal représenté dans les extraits à l'étude semble éviter le registre lyrique autant au plan du contenu qu'á celui de l'expression. 
Tableau 2

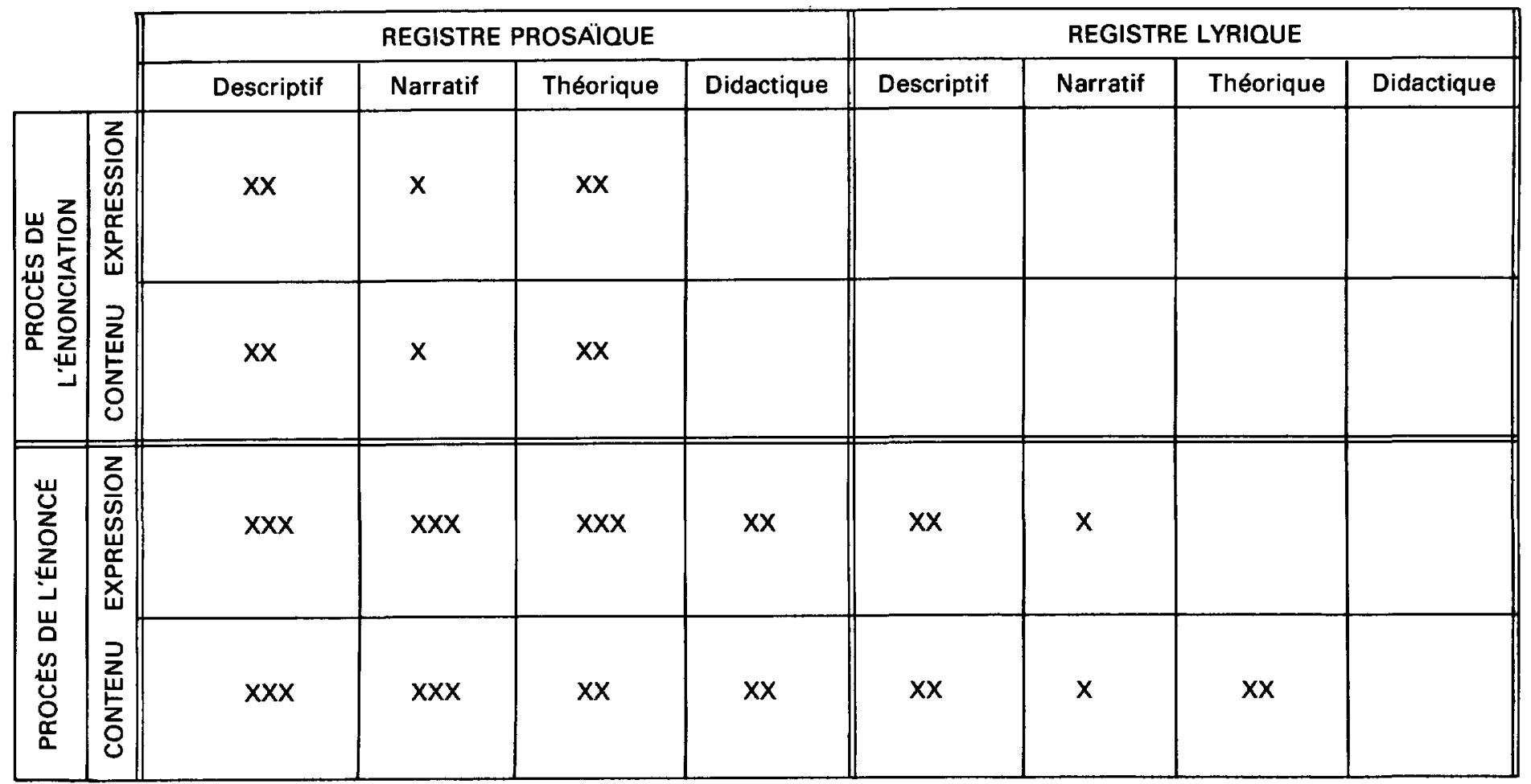


Notre tableau ne met pas assez en évidence un fait intéressant: le hiatus réalisé à quelques reprises entre ces deux plans. Dans le segment narratif:

Ses cheveux, l'espace d'un vingt-quatrième de seconde, sont tous les serpents de l'ancienne tragédie

un contenu imaginaire est rendu dans un style familier. Le discours théorique surtout, lorsqu'il tient de la fiction au plan du contenu, c'est-à-dire d'une construction intellectuelle, conserve une forme usuelle:

ce n'est pas hasard

si nous plaçons les paroles

dans les neiges

Elle (=la pluie) a une façon de mouiller les concepts qui les rend tout gondolés.

Vocabulaire et syntaxe sont tout à fait banals bien que les associations «paroles-neiges» et «mouiller-concepts» soient insolites. La situation inverse est ici moins fréquente:

la neige choit

il fait froid blanc

D'autres extraits manifesteraient une plus grande insistance du registre lyrique ${ }^{37}$ et du discours didactique ${ }^{38}$. Même s'ils négligent certaines particularités, nos résultats reflètent bien l'ensemble du texte. Ce bilan tait par ailleurs l'évolution de l'écriture poétique de Haeck. Un examen minutieux de chacun des temps (des voix) de Polyphonie ferait apparaitre, selon la chronologie, une diminution du registre lyrique au plan de l'expression et une relative stabilité au plan du contenu. II montrerait également une prédominance de plus en plus nette du descriptif et du narratif sur les autres types de discours.

Les quelques phénomènes identifiés ici répondent à un triple souci d'historicité, de lisibilité et d'efficacité. L'écriture de Haeck ne repose ni sur la métaphore ni sur une image englobante. Elle fait souvent référence à l'activité du tisserand et aux effets de la pluie mais ne développe pas de thèmes au sens habituel. Elle reste au contraire essentiellement branchée sur le quotidien:

$J$ 'écris cru, je ne crains par les références

à la vie quotidienne ${ }^{39}$

Les scènes à la plage, sur la rue, en classe ou dans une librairie, scènes d'amour, de travail, d'écriture et de lecture se croisent pour reproduire la quotidienneté. De même la conjonction des divers types de discours, qui comprennent souvent des citations ou des bribes de dialogues, crée un effet d'ambiguïté voulu. On y cherchera en vain la systématicité. Le principe de cette poésie est pluriisotope et excentrique. C'est de loin ce qui fait la difficulté de lecture de Polyphonie, car la simplification syntaxique, l'emploi de prédicats nominaux, l'ellipse et l'absence de ponctuation restent peu fréquents. Définie comme la «passion au ralenti», l'écriture produit, à l'inverse de la littérature avec ses codes et ses normes, une ajoie abruptem. Le plaisir et la beauté semblent tenir alors de l'anarchie et d'une certaine banalisation de la vie qui 
n'est pas sans rappeler le cinéma de Godard. L'auteur de Polyphonie parle, pour sa part, d'une "poéthique de la naïveté» 40 . On assiste à la dissolution de l'angoisse et de l'émotion dans une forme simplifiée. Les marques verbales de la passion à l'œuvre disparaissent chez Haeck alors qu'elles donnent lieu à des formules très recherchées chez d'autres auteurs modernes.

Cette esthétique trouve une partie de son efficacité dans la problématique de l'écriture moderne. Par son aspect prosaique. Polyphonie dénie aux valeurs purement intellectuelles une primauté d'intérêt. L'insertion du quotidien dans la poésie - qui n'en est pas pour autant la transcription - s'oppose à une conception métaphysique de l'art, au caractère fictif de la littérature. La volonté de faire neuf en ayant recours à une rhétorique sinon conventionnelle du moins simplifiée risque de jeter du discrédit sur l'entreprise. Lorsque la forme de l'expression contraste avec la forme du contenu, la praxis est amputée de l'un de ses éléments constituants. Comme toute forme artistique exacerbée perd sa valeur dénonciatrice, la portée critique de la poésie moderne s'est dissoute dans une mode. L'écriture de Philippe Haeck a échappé de justesse à l'écueil du formalisme qui est peut-être le fait d'une déviation gauchiste.

Les amateurs de poésie trouvent leur compte dans le texte de Haeck en dépit de sa monotonie, sans doute parce qu'il y est beaucoup question de l'écriture. Il s'agit encore d'un public spécialisé et non pas des «masses» que cette poésie-propagande souhaite éduquer et mobiliser. Elle veut agir sur les intellectuels d'abord. La littérature reste d'ailleurs une activité plus intellectuelle que pratique et la poésie occupe une place singulière dans le «marché des biens symboliques» ${ }^{41}$. Sa portée ne se compare pas à celle du théâtre ou de la télévision.

La dialectique désir-révolution ne se réalise que par le biais de l'écriture, les termes n'entrant pas en relation directe. De même, l'écrivain a son lieu d'action qui est le discours littéraire. Seul le discours en situation a un effet mesurable. La synthèse entre le sacré et l'histoire, entre la poésie et la réalité n'est rendue possible que par la subjectivité de la langue. L'état en devenir de l'être humain, le coresprit dont parle Philippe Haeck, demeure une réalité poétique.

1. Voir: Jacques Dubois, L'Institution de la littérature, Bruxelles, Editions Labor, 1978.

2. Le «bonheur » peut bien sûr être lié à des questions de vente, de reconnaissance publique, de large diffusion assurée notamment par l'école.

3. Philippe Haeck, Polyphonie. Roman d'apprentissage, Montréal, VLB éditeur, 1978, p. 250

4. Ibid., p. 231.

5. II sera ici essentiellement question de la poésie de Philippe Haeck, rassemblée en 1978 dans Polyphonie. L'emploi de cette notion renvoie inévitablement à une typologie des genres en voie de modification par suite de la pratique de plus en plus libre du discours artistique. Si elle est une mauvaise représentation de la réalité, c'est néanmoins l'indication du rapport hégémonique exercé par l'institution sur les instances de production du texte. II n'est pas insignifiant que Haeck ait 
eu recours à la désignation poèmes. La subversion par l'écriture ne semble pas devoir agir au niveau des étiquettes. Pourtant les textes critiques de l'Action restreinte. De la littérature contestent des notions tout aussi bourgeoises. On remarquera également que la position de classe de l'écrivain ne lui a pas interdit l'accès à l'un des grands médias de l'institution: l'appareil journalistique.

6. Polyphonie, p. 237.

7. Si l'écriture veut défendre une thèse, elle produit d'abord du texte. Dans la pragmatique de la communication, l'analyse de la situation sociale autorise l'examen du rapport production-consommation. Notre objet se trouve ailleurs: non pas dans la fonction externe du texte mais dans son fonctionnement interne. L'étude de ses processus doit nous révéler une problématique en action.

8. Polyphonie, p. 144.

9. Voir le récent numéro d'Études francaises (Vol. 16, nos 3-4), sur le «anifeste poétique/politiquex.

10. Polyphonie, p. 206.

11. Ibid., p. 247.

12. Philippe Haeck, I'Action restreinte. De la littérature, Montréal, Éditions de l'Aurore, 1975, p. 49.

13. Philippe Haeck, Naissances. De l'écriture québécoise, Montréal, VLB Éditions, 1979, p. 319.

14. Polyphonie, p. 37.

15. Polyphonie, p. 36.

16. Ibid., p. 201.

17. Ibid., p. 225.

18. Ibid., p. 155.

19. II existe une imposante bibliographie sur la question du réalisme. On pourra consulter avec profit les travaux de Brecht, de Lukacs, d'Erich Auerbach (Mimesis). de Pierre Barbéris (Aux sources du réalisme...), le numéro 11 de la revue Communications, le numéro 16 de Poétique. Parmi les études faites ici, signalons le numéro 20-21 de Chroniques et l'article de Denis Saint-Jacques: almpossible réalisme» dans Études littéraires, volume 3 numéro 1, avril 1970, pp. 9-19.

20. Philippe Van Tieghem, Dictionnaire des Littératures, Paris, P.U.F., 1968, p. 3264.

21. J.A. Cuddon, A Dictionary of Literary Terms, London, Andre Deutsch, 1977 c, p. 542.

22. Naissances, p. 328.

23. Polyphonie, p. 120.

24. Naissances, p. 323

25. Ibid., p. 318.

26. Ibid., p. 367 .

27. Ibid., p. 377.

28. Groupe $\mu v$, Rhétorique de la poésie, Bruxelles, Editions Complexe, 1977.

29. Cf. Louis Hjelmslev, Prolégomènes à une théorie du langage, Paris, les Editions de Minuit, 1968, pp. 65-79.

30. Polyphonie, p. 103.

31. Ibid., p. 57.

32. Théorie de la littérature (textes des Formalistes russes réunis, présentés et traduits par Tzvetan Todorov), Paris, Seuil, 1965, p. 289.

33. Polyphonie, p. 114.

34. lbid., pp. 13-14.

35. Ibid., pp. 33-34. Ce texte revêt un caractère particulier comme l'indique un passage de Naissances, pp. 361-362.

36. Ibid., p. 215.

37. Voir notamment Polyphonie, pp. 15, 16, 99.

38. Voir notamment Polyphonie, pp. 153, 207, 236, 299. 
392 VOIX ET IMAGES, VOL. VI, NO 3

39. Ibid., p. 139.

40. Ibid., p. 274.

41. Pierre Bourdieu, "Le marché des biens symboliques", L'Année sociologique, 3e série, 1971, pp. 49-126. 